\title{
EMIGRAÇÃO E TRANSFERÊNCIAS MONETÁRIAS COMO ESTRATÉGIAS DE ADAPTAÇÃO ÀS SECAS NO SERIDÓ POTIGUAR
}

\author{
Out-migration and cash transfers as a livelihoods adaptation \\ to the droughts in the Seridó Potiguar
}

\author{
Isac Alves Correia* \\ Ricardo Ojima** \\ Alisson Flávio Barbieri***
}

\begin{abstract}
Resumo. A partir de um survey urbano realizado em 2017, com representatividade para a região do Seridó Potiguar (Rio Grande do Norte), no Semiárido Nordestino, o objetivo desse artigo é entender como os programas sociais e as remessas de migrantes têm-se constituído em estratégias centrais de adaptação às secas. A metodologia consiste em estatísticas descritivas, testes estatísticos de representatividade amostral e de diferenças entre proporções. Os principais resultados mostram que as remessas enviadas pelos emigrantes foram mais presentes para famílias com níveis de renda mais baixos, de até dois salários mínimos per capita, representando um apoio fundamental para as famílias na região de origem. Os programas sociais também foram mais marcantes em famílias de menores rendas. A concentração da população na zona urbana parece interligada a esses processos, apresentando determinadas vantagens em termos de estratégias domiciliares com a busca por ocupações no mercado de trabalho urbano para incrementar a renda familiar.
\end{abstract}

Palavras-chave: migração; seca; transferência de renda.

Abstract. Based on an urban survey undertaken in 2017 and involving more than one thousand households in the Seridó Potiguar, in the Brazilian Semiarid, this paper aims to analyze e objective of this paper is to understand how governmental social programs and migrant's remittances have become a

\footnotetext{
Doutorando em Demografia pelo Centro de Desenvolvimento e Planejamento Regional (Cedeplar/UFMG). Belo Horizonte - MG, Brasil. E-mail: isaccorreia@cedeplar.ufmg.br. Orcid: 0000-0001-9495-2325.

** Professor Adjunto do Departamento de Demografia e Ciências Atuariais da Universidade Federal do Rio Grande do Norte (DDCA/UFRN). Natal - RN, Brasil. E-mail: ricardo.ojima@gmail. Orcid: 0000-0002-7472-4285.

${ }^{* * *}$ Professor Adjunto do Departamento de Demografia no Centro de Desenvolvimento e Planejamento Regional (Cedeplar/UFMG). Belo Horizonte - MG, Brasil. E-mail: barbieri. alisson@gmail. Orcid: 0000-0002-1133-1089.
} 
key household adaptation strategies to droughts. The methodology consists in descriptive statistics, and, tests of statistical sampling and differences among proportions. The results show a higher prevalence of remittances sent by emigrants to for families with lower income levels in the origin (until two minimum wages per capita), which represents a key component supporting for these families. Cash transfer programs were more important for families of smaller per capita incomes. This finding seems related to the concentration of population in the urban zone, although some advantages seem to exist regarding household strategies regarding the allocation of one or some of their members in urban labor markets in order to increase family income.

Keywords: migration; drought; cash transfer.

\section{Introdução ${ }^{1}$}

Durante décadas, a literatura se apropriou do fenômeno da seca enquanto elemento de explicação das condições sociais do Nordeste (Melo Neto, 1965²; Queiroz, 1930; Ramos, 1938) e dos fluxos migratórios da região (Furtado, 1959; Martine, 1994; Araújo, 2012). Embora esse esforço tenha sido relevante para entender a dinâmica migratória da região e suas implicações, a relação entre esse fenômeno e as estiagens permanece inconclusiva. Foram poucas as pesquisas que exploraram as estratégias familiares de adaptação e convivência com tais condições.

O objetivo desse artigo é entender como transferências monetárias poderiam significar uma estratégia de adaptação às secas em uma região do semiárido brasileiro. Parte-se de uma pesquisa domiciliar urbana realizada entre janeiro e fevereiro de 2017 com amostra representativa para a região do Seridó Potiguar. Foram coletadas informações de 1.064 domicílios nessa região formada por 17 municípios, a saber: Caicó, Ipueira, Jardim de Piranhas, São Fernando, São João do Sabugi, Serra Negra do Norte, Timbaúba dos Batistas, Acari, Carnaúba dos Dantas, Cruzeta, Currais Novos, Equador, Jardim do Seridó, Ouro Branco, Parelhas, Santana do Seridó e São José do Seridó. Conforme a Figura 1, tais municípios correspondem às microrregiões do Seridó Ocidental e Oriental, no Rio Grande do Norte.

O Seridó é exemplar para entender a interação entre as estratégias de adaptação das famílias, particularmente a migração e o fenômeno da seca no semiárido nordestino. Nessa região predominam altas temperaturas, baixa fertilidade do solo, regime de chuvas irregulares e uma população que

1 Uma versão preliminar desse trabalho foi apresentada no XXI Encontro Nacional de Estudos Populacionais (ABEP), Poços de Caldas/MG, 2018.

2 Publicada pela primeira vez em 1956 pela editora José Olympio em volume intitulado Duas águas. 
depende basicamente de atividades agropastoris. A região possui diversos polos de desertificação, tendo enfrentado, no biênio 2011-2012, uma das maiores estiagens dos últimos 30 anos e a maior crise hídrica dos últimos 50 anos. Além disso, trata-se de uma região populosa do semiárido, com 216 mil habitantes e cerca de $85 \%$ da população vivendo na zona urbana, o que pode fornecer elementos analíticos importantes para entender sinergias entre o rural e o urbano em relação as estratégias domiciliares (Barbieri et al., 2018; Correia, Ojima, 2018).

Figura 1 - Localização do Seridó Potiguar, Rio Grande do Norte e municípios das microrregiões do Seridó Ocidental e do Seridó Oriental - 2010

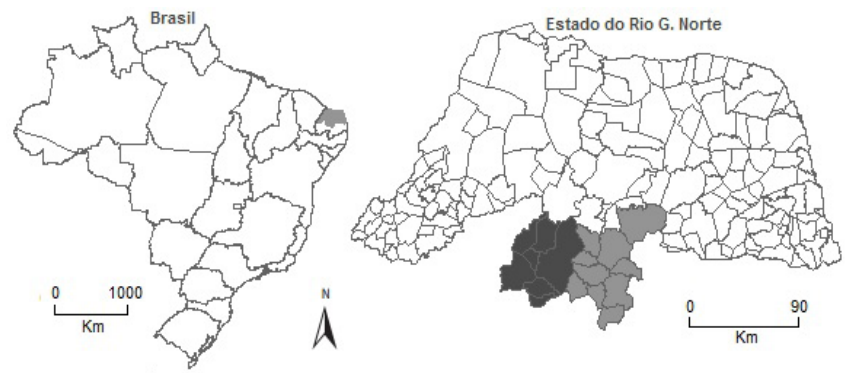

Seridó Potiguar

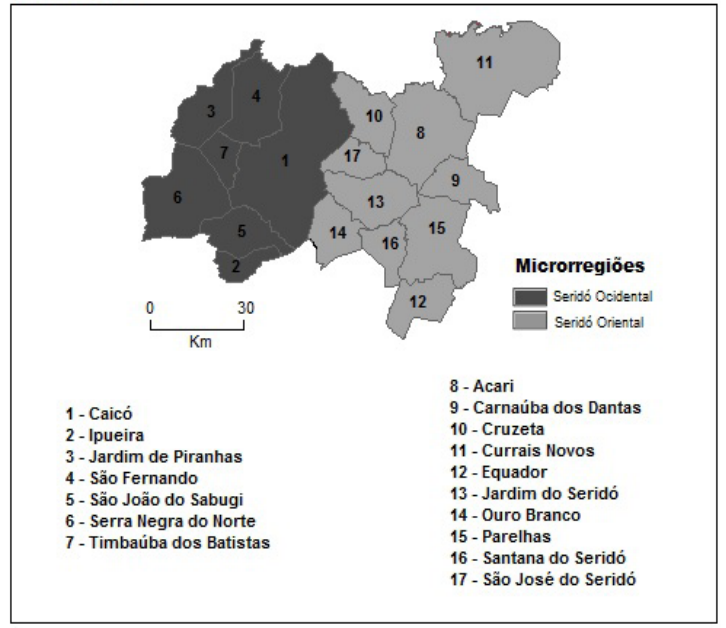

Fonte: IBGE, Malha Municipal Digital (2010).

A metodologia adotada no trabalho consiste em estatísticas descritivas, testes estatísticos de representatividade amostral e de diferenças entre proporções. O artigo está estruturado em três seções. A primeira apresenta uma revisão da literatura sobre migração e seca no semiárido, na segunda são apontados e discutidos os resultados e na última seção são feitas as 
considerações finais. O estudo conclui que as transferências monetárias podem se converter em ferramentas importantes na adaptação das famílias às secas no Seridó Potiguar, com o auxílio de políticas públicas que facilitem a incorporação desses recursos na economia local por meio da conversão em investimentos produtivos.

\section{Migração, seca e vulnerabilidade no Semiárido Brasileiro e no Seridó Potiguar}

A região Nordeste como um todo é tradicionalmente classificada como expulsora de população, seja por fatores ambientais ou por indicadores socioeconômicos e/ou regionais inferiores às áreas de atração dessas populações (Oliveira, 2008; Fusco, Duarte, 2010). Embora sempre destacadas nos estudos, quase nunca as questões ambientais são colocadas em posição central das análises (Ojima, 2013).

Estudos recentes recortando essa região mostram que mais de $75 \%$ dos municípios possuem $\mathrm{TLM}^{3}$ negativas, inclusive no Seridó Potiguar que está integralmente inserido no semiárido. Isso significa que a maior parte dessa área tem características de expulsão de população que, notadamente, se associa às desigualdades regionais (Correia, Ojima, 2018; Correia, 2018). Por outro lado, existem particularidades dentro desses estados no que diz respeito à dinâmica migratória. As TLM negativas mais altas se concentram na porção semiárida dos estados, diferentemente do que acontece com os municípios do entorno. Os municípios do norte de Minas Gerais e os municípios costeiros dos estados apresentam taxas positivas diferenciando-os da porção semiárida.

Algumas pesquisas assinalam uma queda de cerca de $80 \%$ na produção agrícola do semiárido em períodos de secas (Khan et al., 2005), intensificando os processos migratórios (Barbieri, 2011) e reconfigurando as situações de vulnerabilidade no território (Adger et al., 2015). Por outro lado, não há consenso na literatura, tanto na discussão se a migração seria uma alternativa de adaptação ou se ela contribuiria para reiterar cenários de vulnerabilidade (Barbieri, 2011) quanto na intensificação desses movimentos populacionais motivados por mudanças ambientais (Adger et al., 2015; Koubi, Stoll, Spilker, 2016). Além das perdas na produção e na receita agrícola, o efeito das estiagens sobre o emprego (Khan et al., 2005) podem contribuir para realçar o modo de

\footnotetext{
3 A Taxa Líquida de Migração (TLM) é calculada por meio da diferença entre o número de imigrantes e emigrantes de data-fixa dividida pela população total do município no final do período. Para facilitar a interpretação, a TLM comumente é multiplicada por 100. Assim, a TLM corresponde ao efeito líquido da migração no crescimento da população do município. A TLM, caso seja positiva, indica a proporção da população observada no segundo censo resultante do processo migratório; e, caso negativa, indica a proporção em que a população seria acrescida na ausência de migração. Essa interpretação da TLM enfatiza o papel da migração (numerador, SM) sobre o crescimento populacional (denominador).
} 
vida das cidades que se estende para além das metrópoles (Monte-Mór, 2006; Baeninger, 2012) integrando as atividades agrícolas e não-agrícolas (Vanwey, Guedes, D'antona, 2012) e a busca por serviços públicos (Ojima, 2013).

Tanto a mobilidade, por meio das remessas dos migrantes para a região de origem, quanto os programas de transferência de renda, poderiam reduzir a vulnerabilidade dos domicílios à seca ao fornecer um incremento na renda das famílias. A mobilidade, por sua vez, possibilita o acesso a mercados de crédito urbano, empregos e outras fontes de renda que constituem formas de diversificar os riscos a potenciais choques e oscilações econômicas, incluindo os de natureza ambiental. Essas transferências, entretanto, podem causar dependência por parte da região de origem ao limitar a capacidade de adaptação das famílias beneficiárias à mudança e serem convertidos em atividades menos ou sem nenhum intuito produtivo. Por outro lado, estudos têm mostrado a importância das transferências monetárias e da renda não agrícola como forma de melhorar as estratégias de sobrevivência das famílias, especialmente no contexto amazônico (Vanwey, D’antona, Brondízio, 2007; Sherbinin et al., 2008; Duo et al., 2017).

Muito embora a mobilidade possa constituir-se em uma importante estratégia de sobrevivência para as famílias, uma maior mobilidade tem consequências e desafios para as instituições, já que pode representar um mecanismo de gerar novos riscos e vulnerabilidades. De forma complementar, os migrantes que saem do Semiárido em direção às grandes metrópoles podem ser, em muitos dos casos, mais vulneráveis do que os que lá já residem há mais tempo, embora o estabelecimento de redes e capital social possa reduzir o grau de exposição desses indivíduos ao risco e suas vulnerabilidades (Black et al., 2011; Adger et al., 2015).

Bourdieu definiu o capital social como "o agregado dos recursos efectivos ou potenciais ligados à posse de uma rede durável de relações mais ou menos institucionalizadas de conhecimento ou reconhecimento mútuo" (Bourdieu, 1985 , p. 248). O compartilhamento de informações sobre linhas de crédito, técnicas de cultivo e empréstimos na região de origem são exemplos dessa forma de capital que podem reduzir a vulnerabilidade da população às estiagens no Seridó Potiguar.

É necessário, no entanto, construir um debate mais amplo que incorpore tanto a vulnerabilidade das famílias às secas nas regiões semiáridas, as estratégias de adaptação considerando a multiplicidade das escalas, quanto as implicações para as regiões receptoras de migrantes. Nesse sentido, embora a mobilidade em seu sentido mais amplo e a urbanização nos municípios do Semiárido tenham algumas consequências negativas, podem ser alternativas 
para reduzir a vulnerabilidade das famílias com o engajamento em atividades urbanas (Vanwey, Guedes, D’antona, 2012; Ojima, 2013).

A partir da dinâmica da população urbana do Seridó Potiguar e as especificidades do fenômeno das secas na região, será possível extrair conexões com as estratégias de sobrevivência das famílias. Nesse sentido, as respostas às pressões ambientais e/ou econômicas podem ter estreita relação com a diversificação da renda por meio da renda não agrícola e o processo de concentração da população na zona urbana desses municípios. Ou seja, mesmo que representem ganhos em economia de escala na oferta de serviços básicos, por exemplo, podem intensificar processos de degradação e demanda por serviços ecossistêmicos como constatado para o contexto amazônico (Sherbinin et al., 2008; Vanwey, Guedes, D'antona, 2012; Ojima, 2013).

As dinâmicas rurais, embora ainda pouco exploradas na região, parecem estar crescentemente interligadas ao meio urbano pela dupla residência de seus membros ou pela oferta de trabalho de seus moradores na cidade (Vanwey, Guedes, D’antona, 2012; Barbieri, Pan, 2013; Lombardi, Guedes, Barbieri, 2015). Estudos que privilegiam a análise dessas regiões emissoras de fluxos migratórios e as sinergias com as regiões receptoras desses migrantes são cada vez mais importantes para ajudar na compreensão dessas estratégias de sobrevivência das famílias e dos desafios institucionais que as mudanças ambientais impõem. É nesse recorte analítico que este trabalho se insere. Desta forma, busca-se compreender as conexões entre locais de origem e destino dos fluxos migratórios internos e entender o papel das políticas sociais como mecanismos relevantes para adaptação às condições sociais e ambientais em contextos urbanos.

\section{Dados e métodos}

A pesquisa é resultado do projeto "Vulnerabilidade e Adaptação no Nordeste Brasileiro: Perspectivas Locais e Regionais sobre a Urbanização no Seridó Potiguar" financiado pela Rede Brasileira de Pesquisas sobre Mudanças Climáticas Globais (RedeCLIMA) e Conselho Nacional de Desenvolvimento Científico e Tecnológico (CNPq). Esse trabalho representa uma das primeiras tentativas de preencher algumas lacunas das pesquisas populacionais e dos estudos que tentam entender o fenômeno migratório e sua relação com a seca no semiárido brasileiro, sobretudo no recorte territorial específico.

O trabalho de campo ocorreu entre os meses de janeiro e fevereiro de 2017 com uma amostra de 1.064 domicílios urbanos representativas para o Seridó Potiguar. As entrevistas ocorreram dentro de um período de três semanas com tempo médio de 35 minutos por entrevista. Os municípios selecionados na amostra e as respectivas entrevistas foram distribuídos conforme a Tabela 1. 
A amostra foi probabilística e representativa da população do Seridó Potiguar segundo a probabilidade de seleção em cada um dos estágios de seleção (estágio 1: município; estágio 2: setor censitário; e estágio 3: domicílio).

Tabela 1 - Seridó Potiguar: entrevistas realizadas por município e população urbana estimada, 2017

\begin{tabular}{lcccc}
\hline Município & $\begin{array}{c}\text { Questionários } \\
\text { aplicados }\end{array}$ & $\begin{array}{c}\text { Total de pessoas } \\
\text { nos domicílios } \\
\text { (amostra) }\end{array}$ & $\begin{array}{c}\text { No de domicílios } \\
\text { (expandidos) }\end{array}$ & $\begin{array}{c}\text { No de pessoas } \\
\text { (expandidas) }\end{array}$ \\
\hline Acari & 75 & 245 & 6.872 & 22.139 \\
Caicó & 359 & 1.220 & 11.272 & 38.170 \\
Cruzeta & 85 & 260 & 6.300 & 19.900 \\
Currais Novos & 306 & 1.040 & 8.110 & 27.340 \\
Ipueira & 30 & 100 & 11.691 & 38.971 \\
Parelhas & 180 & 607 & 7.103 & 24.534 \\
São João do Sabugi & 29 & 82 & 7.920 & 22.394 \\
\hline Total & 1.064 & 3.554 & 59.268 & 193.448 \\
\hline
\end{tabular}

Fonte: Survey Seridó Potiguar, 2017. 1. A população base das projeções para a população urbana da região foi retirada do Censo Demográfico 2010 do IBGE. Em 2010 segundo o IBGE a população urbana dessa região era de 182.824 habitantes.

Como a pesquisa tratou de uma investigação urbana, no estágio 1 optou-se pelo método de amostragem do tipo aleatória proporcional ao número de setores urbanos do município. No estágio 2 o método foi aleatório simples com um N fixado em $20 \%$ do número de setores urbanos no município. Isso permitiu uma maior representatividade para os municípios com maiores números de setores urbanos. No estágio 3, por sua vez, foi adotada a amostragem aleatória estratificada, em que os estratos foram definidos como: 1) domicílios com renda média inferior ou igual à $R \$ 300,00$ mensais para as pessoas de 10 anos e mais (estrato 1) e 2) domicílios com renda média superior à $R$ \$300,00 mensais para as pessoas de 10 anos e mais.

\section{Conceitos, variáveis e categorias de análise}

O migrante, nesse artigo, apresenta dois conceitos. No primeiro, entende-se por migrante de data fixa o indivíduo que residia em um município diferente do de residência atual há exatamente cinco anos anteriores à data de referência do Censo, ou seja, o migrante captado no quesito de data-fixa dos Censos Demográficos. Na segunda, o migrante do último sexênio é o indivíduo que, no período de 2010 a 2016, residiu em um município diferente do de residência no período em que o survey foi realizado. Essa informação foi captada pela pergunta "D1 - Alguém do domicílio emigrou para outro 
município entre 2010-2016?", em que serão distinguidos os domicílios com emigrante e domicílios sem emigrante.

A escolha do período específico de 2010 a 2016 é justificável pelo fato de coincidir com o período de ocorrência de estiagem e aumento da temperatura média anual na região que, segundo alguns pesquisadores, se intensificou nos últimos anos (Souza et al., 2014). Estas mudanças reproduzem situações específicas de vulnerabilidade, notadamente expressas na perda de status socioeconômico das famílias e podem ter influência na diversificação de suas estratégias de sobrevivência, dadas essas situações de risco (Carr et al., 2008; Barbieri, Carr, Bilsborrow, 2009; Ojima, 2013).

Também foi perguntado se "essas pessoas mandam dinheiro ou algum tipo de ajuda regularmente para quem mora nessa casa?", que figurará como parte das transferências monetárias para as famílias na região de origem. Outros tipos de transferências monetárias analisadas dizem respeito aos Programas de Transferência de Renda (PTR), com o intuito de avaliar o papel destas políticas para gerar condições para adaptação a situações de vulnerabilidades específicas, como poderia ser o aumento da emigração. Para captar essas informações, foi perguntado: "recebe algum benefício social?". Para os entrevistados beneficiários dos PTR foi perguntado: "acha que se não recebesse esses benefícios você ou algum morador dessa casa teria mudado de cidade nesses últimos 5 anos?".

Além da renda domiciliar per capita, analisou-se a renda proveniente da atividade não agrícola, que diz respeito à renda domiciliar per capita (exceto a renda proveniente de atividades agrícolas), cujas categorias adotadas são: i) Até 1⁄2 salário mínimo 4 ;ii) 1⁄2 a 1 salário mínimo; iii) 1 a 2 salários mínimos; iv) 2 a 3 salários mínimos; v) 3 a 4 salários mínimos; e vi) 4 salários mínimos e mais. Nesse sentido, tanto as respostas econômicas por meio da diversificação da renda (Carr et al., 2008; Barbieri, Carr, Bilsborrow, 2009) quanto a vulnerabilidade das famílias às mudanças ambientais (Gilbert, Mcleman, 2010; Massey, Axinn, Ghimire, 2010) serão analisadas.

Além disso, a escolha do período específico de 2010-2016 é justificada pelo fato de coincidir com um período de ocorrência de eventos climáticos extremos (secas e aumento da temperatura média anual) que sugerem mudanças nas estratégias de sobrevivência da população na região. Estas mudanças podem reproduzir situações específicas de vulnerabilidade expressas na perda de status das famílias com a queda na renda agrícola e que podem ter influência na diversificação de estratégias de sobrevivência dessas famílias (Ojima, 2013), dadas as situações de risco. Para analisar a relação entre a seca e a migração como estratégia de adaptação aos meios de subsistências,

4 Com base no valor do salário mínimo de R\$ 937,00, vigente em 2017. 
perguntou-se: "já pensou em se mudar por causa da seca?" e, de forma complementar, foram feitas comparações para os domicílios com emigrante e domicílios sem emigrante.

\section{Análise dos dados}

Os dados do Survey foram analisados por meio de estatísticas descritivas e a estatística Deff (Design Effect) para mensurar o impacto do plano amostral complexo sobre a variabilidade das informações. O Deff é calculado por meio da razão entre a variância estimada do plano amostral e a variância estimada supondo amostra aleatória simples. Dessa forma, o Design Effect - que é apresentado para apontar o impacto de não considerar o desenho amostral complexo - é obtido por meio da raiz quadrada do Deff. Quanto mais distante ele for da unidade, mais imprecisa será a estimativa que não leve em consideração o desenho amostral complexo (Cameron, Trivedi, 2005).

Foram utilizados os testes de diferenças entre duas proporções, o Qui-quadrado $\left(X^{2}\right)$, para comparar as frequências esperadas e observadas. O teste Qui-quadrado informa se dois grupos se comportam de forma semelhante ou diferente ao se analisar a significância estatística da diferença nas frequências relativas desses grupos. Dessa forma, com um $p$-valor menor que 0,05 , rejeita-se a hipótese nula de que as diferenças entre as categorias das variáveis selecionadas não são estatisticamente significativas para domicílios com emigrantes em relação aos domicílios sem emigrantes. Considerou-se, ainda, a exigência de que haja independência entre os grupos, aleatoriedade e a existência de um número de observações relativamente grande (Pearson, 1990).

\section{Vulnerabilidade, principais motivos da emigração e transferências monetárias no Seridó Potiguar}

Os municípios do Rio Grande do Norte, de um modo geral, passaram por uma situação difícil entre 2011 e 2017 devido aos baixos índices pluviométricos, especialmente os municípios do Seridó, que é a região mais seca do estado. As estiagens provocaram prejuízos financeiros de R\$ 4 bilhões (72,30\% na agricultura; 27,70\% da pecuária do estado) nesse período, de acordo com os dados da Secretaria de Estado da Agricultura, da Pecuária e da Pesca (SAPE). Além disso, um relatório divulgado em 2017 pelo Instituto de Gestão das Águas (IGARN) mostrou que os principais açudes do Seridó tiveram suas capacidades comprometidas (RN, 2017), afetando o abastecimento de água e os meios de subsistência das famílias não apenas nas atividades agrícolas, mas também na economia urbana devido aos racionamentos e rodízios de abastecimento de água e utilização de carros pipa e aquisição de água potável em estabelecimentos comerciais. 
Assim, quando perguntados sobre a migração como uma estratégia para fugir dos períodos de estiagem atual e do passado, cerca de $23 \%$ dos casos a pessoa de referência do domicílio respondeu já ter pensado em se mudar por conta da seca. Nos domicílios com emigrante (26,4\%), esse percentual foi ligeiramente maior em relação aos domicílios sem emigrante $(22,8 \%)$ como mostra a Tabela 2. A estatística do teste Qui-quadrado mostrou que essas proporções possuem diferenças estatisticamente significativas. Isso possivelmente pode ser explicado por haver a possibilidade de outras estratégias de adaptação além da migração nesses casos (Bardsley, Hugo, 2010). Assim, a migração pode ser uma resposta adiada até que todas as alternativas in situ sejam esgotadas (Nawrotzki, Dewaard, 2016). Há que se considerar também que, especialmente no caso do semiárido nordestino, a seca possibilita múltiplas respostas (Gray, Mueller, 2012) devido os efeitos sobre os meios de subsistência e sobrevivência dos indivíduos serem de início lento, contribuindo para respostas de curto prazo.

Tabela 2 - Seridó Potiguar: distribuição relativa (\%) dos domicílios urbanos, com e sem emigrantes, segundo a percepção sobre a decisão de migração induzida pela seca, 2017

\begin{tabular}{lcccccc}
\hline & \multicolumn{5}{c}{ Domicílios } & \\
\cline { 2 - 5 } $\begin{array}{l}\text { Já pensou em se } \\
\text { mudar por causa } \\
\text { da seca? }\end{array}$ & \multicolumn{5}{c}{ Com emigrante } & \multicolumn{2}{c}{ Sem emigrante } & \multirow{2}{*}{ I. C - 95\% } & Deff \\
\cline { 2 - 5 } & $\mathbf{N}$ & $\%$ & $\mathbf{N}$ & $\%$ & & \\
\cline { 2 - 6 } Sim & 2.452 & 26,4 & 11.403 & 22,8 & $(8.035-19.674)$ & 3,1 \\
Não & 6.443 & 69,4 & 38.210 & 76,4 & $(29.192-47.229)$ & 4,9 \\
Não sabe & 390 & 4,2 & 370 & 0,7 & $(-151-1.671)$ & 1,8 \\
\hline Total & 9.285 & 100,0 & 49.983 & 100,0 & Qui-quadrado $=$ & $826,07^{* * *}$ \\
\hline
\end{tabular}

Fonte: Survey Seridó Potiguar, 2017. ${ }^{* *} p<0,01,{ }^{*} p<0,05,{ }^{*} p<0,1$ para teste $X$-squared de diferenças entre proporções. $n=n$ o de observações pequeno.

Outra observação importante é que, no caso particular da seca nessas regiões, ao longo de sua experiência migratória com retenção populacional para áreas mais dinâmicas do país anexo aos índices de desigualdade e pobreza além da média nacional, leva-se a crer que a vulnerabilidade das instituições e dos domicílios pouco tem contribuído para medidas de adaptação in situ efetivas. Apesar das mudanças nas tendências migratórias com perda da expressividade dos fluxos de longa distância, o Nordeste continua perdendo um contingente populacional significativo para outras regiões do país (Correia, 2018). As TLM tiveram um impacto mais significativo sobre as populações dos municípios do semiárido vis-à-vis os municípios de estados do Nordeste e Minas Gerais que não compõem esse recorte territorial. Por outro lado, as respostas de domicílios 
com e sem emigrante referem-se a pessoas que permanecem no município de origem, o que pode ser uma alternativa tão estratégica quanto a própria saída da região (Correia, Ojima, 2018).

O período recente de seca que durou mais de seis anos pode ter dificultado o abastecimento de água para o consumo doméstico sem apresentar impactos diretos sobre as condições vitais, mas inviabilizou atividades econômicas e agropastoris que são importante fonte de renda de famílias da região ou dificulta o acesso a outros meios de subsistência, alimentos, empregos etc. O retardamento dos períodos de chuvas e ocorrências de baixos índices pluviométricos na região podem ter contribuído para acentuar os processos migratórios e/ou oportunizar outras mudanças nas estratégias de sobrevivência das famílias, seja de forma indutiva ou por pressões de caráter endógeno (Barbieri et al., 2010; Barbieri, 2011; Ojima, 2013), com danos para a prática da agricultura de sequeiro na região do Seridó, com descapitalização de camponeses e afetando negativamente nos processos decisórios dos produtores rurais com queda na produção e na demanda por trabalho agrícola (RIO GRANDE DO NORTE, 2017).

Assim, as secas podem estimular o processo migratório de forma endógena para os domicílios cujas estratégias para mitigar essas mudanças falharam (Barbieri et al., 2010). Por outro lado, em situações em que os domicílios dispõem de maiores oportunidades de escolhas, é possível que essa dualidade entre estratégias de adaptação in situ e mobilidade não exista (Bardsley, Hugo, 2010). Determinadas situações de risco podem representar uma oportunidade para os domicílios estabelecerem relações diretas e indiretas com mercados urbanos (Sherbinin et al., 2008), como a dupla residência que permitiria acesso a empregos urbanos e serviços básicos.

A renda per capita para os domicílios com emigrante é, em média, $\mathrm{R} \$$ 650,80 , e para domicílios sem emigrante de $R \$ 782,75$. Portanto, nos domicílios sem emigrante a renda domiciliar per capita é maior do que nos domicílios com emigrante. Os domicílios com renda mais elevada, portanto, dependem menos que um dos moradores migre, devido ao acesso que esses domicílios teriam a outras estratégias de adaptação (Warner et al., 2009; Bardsley, Hugo, 2010).

Por outro lado, o Gráfico 1 mostra que nos domicílios com renda domiciliar per capita entre 1/2 e 1 salário mínimo a participação de domicílios com emigrantes é maior. Embora uma parcela da população possa considerar a migração como uma estratégia de adaptação (Bardsley, Hugo, 2010), seja por questões puramente econômicas e/ou ambientais, a decisão de migrar envolve uma combinação de uma série de motivações, desde econômicas às aspirações individuais, mas também por influências dos custos intervenientes (Lee, 1966; 
Hugo, 1996) e, nesse caso, de outras estratégias de adaptação disponíveis aos indivíduos ou domicílios (Warner et al., 2009; Nawrotzki, Dewaard, 2016).

Gráfico 1 - Seridó Potiguar: distribuição relativa (\%) dos domicílios urbanos, com e sem emigrantes, segundo a renda domiciliar per capita não agrícola e total (em salários mínimos), 2017

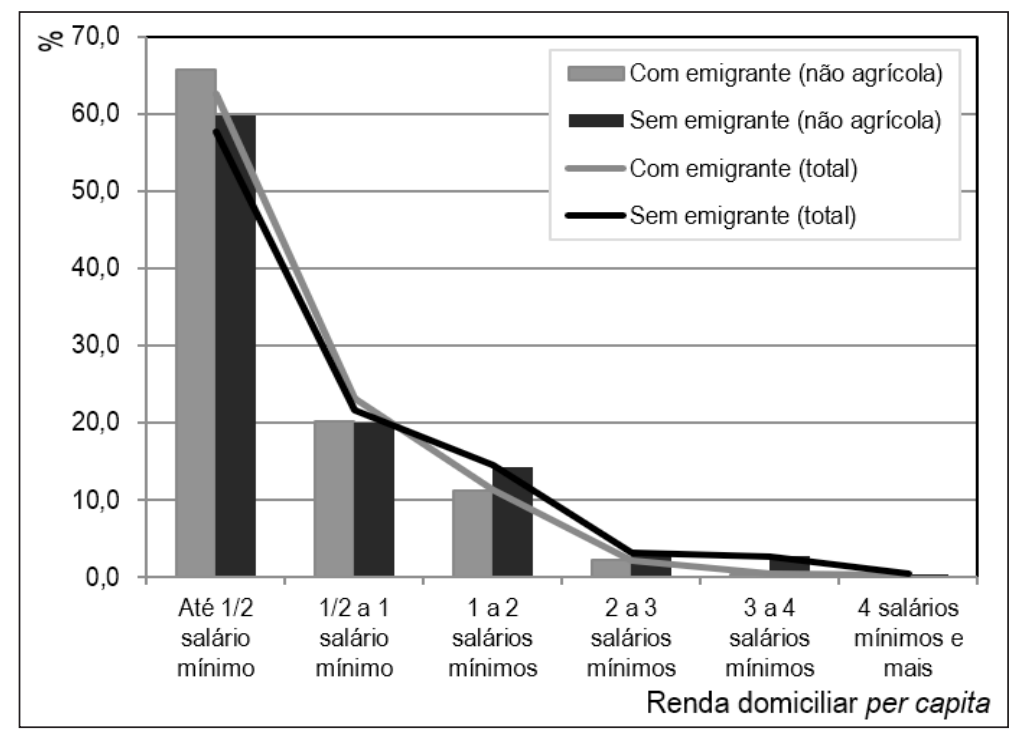

Fonte: Survey Seridó Potiguar, 2017.

Analisando a renda per capita dos domicílios, para aqueles com renda não agrícola domiciliar per capita entre 1/2 e 1 salário mínimo e, especialmente, os de 4 salários mínimos e mais, os domicílios com e sem emigrantes não se distanciam muito. Para os domicílios com renda domiciliar per capita de até 1/2 salário mínimo, contudo, a renda não agrícola tem uma participação relativa importante na composição da renda total, com uma maior participação para domicílios com emigrante, sugerindo uma combinação de estratégias no nível do domicílio com a migração e o incremento da renda com atividades não agrícolas (Sherbinin et al., 2008; Barbieri, Carr, Bilsborrow, 2009; Vanwey, Guedes, D'antona, 2012; Ojima, 2013).

Outra questão importante é que mais da metade dos domicílios com emigrantes e que recebem remessas $(56,1 \%)$ está concentrada nos níveis de renda per capita de até 2 salários mínimos. O efeito das remessas na renda familiar, entretanto, ainda não pode ser comprovado haja vista estar-se comparando a renda per capita dos domicílios com emigrantes com os domicílios sem emigrantes, dadas as limitações dessa pesquisa. No entanto, as remessas podem ter um papel importante no processo de adaptação das 
famílias às secas na região do Seridó Potiguar, especialmente para as que estão nesses níveis de renda per capita.

Essas remessas podem tornar as regiões de origem dos migrantes cada vez mais dependentes das regiões de destino, sendo altamente vulneráveis a crises externas (Corbin, Aragón, 2015). Portanto, é necessário recorrer também a estratégias de convivência e adaptação por meio de políticas públicas que considerem estas particularidades, mas que também incorporem a complementariedade das escalas. Essas políticas, ao facilitarem a penetração das transferências monetárias na economia local por meio de investimentos produtivos, podem contribuir para uma redução da dependência de recursos por parte desses indivíduos.

No que diz respeito aos motivos da migração declarados pelos entrevistados de domicílios com emigrantes, os principais resultados são relacionados à busca por trabalho $(37,5 \%)$, estudo $(36,7 \%)$ ou questões pertinentes à família $(18,9 \%)$, incluindo o casamento ou conflitos intra-domiciliares. Conforme mostra a Tabela 3, 23\% das famílias com pelo menos um dos membros vivendo em um município diferente do agregado domiciliar recebem remessas desses indivíduos. A maior parte dos moradores que emigraram e que enviam remessas para suas famílias na região de origem emigraram por motivo de trabalho (62,5\%), seguidos pelos motivos de estudo $(27,1 \%)$ e de questões relacionadas à família (5,8\%), sinalizando a importância da migração para o incremento da renda dessas famílias (Carr et al., 2008; Barbieri, Carr, Bilsborrow, 2009). Os valores Deff mostram pouca influência do desenho amostral sobre as estimativas, indicando consistência nos resultados. A estatística do teste Qui-quadrado, por sua vez, mostrou que existem diferenças estatisticamente significativas entre essas proporções.

Em se tratando do destino dos emigrantes, a Tabela 3 mostra que a maior parte $(68,3 \%)$ dos indivíduos que não enviam remessas para suas famílias na origem são indivíduos que migraram para municípios da própria região Nordeste, exclusive os municípios do estado do Rio Grande do Norte. Os indivíduos que enviam remessas para o domicílio de origem, por sua vez, migraram para municípios distribuídos entre a região Nordeste $(39,4 \%)$ e outras regiões do país (36\%). A literatura tem indicado que a mobilidade da seca em outras regiões tem sido essencialmente de caráter temporário (Gray, Muller, 2012). Convergente à literatura, os resultados da pesquisa indicam que a distância entre as regiões de origem e de destino dos migrantes ${ }^{5}$ ajuda a explicar a presença de remessas na renda das famílias na origem. A presença de remessas nesses domicílios pode

\footnotetext{
Uma limitação da pesquisa é que ela não captou o sexo e a idade do emigrante, o que pode ter influência sobre o envio de remessas para a região do Seridó.
} 
atenuar situações de riscos e de vulnerabilidade, diversificando a renda das famílias (Sherbinin et al., 2008; Vanwey, Guedes, D’antona, 2012).

Tabela 3 - Seridó Potiguar: distribuição relativa (\%) dos emigrantes com origem em domicílios urbanos, segundo o envio de remessa, o motivo e o destino da migração, 2017

\begin{tabular}{|c|c|c|c|c|c|c|}
\hline \multirow{2}{*}{ Motivo da migração } & \multicolumn{4}{|c|}{ Envio de remessa } & \multirow{2}{*}{ I. C- $95 \%$} & \multirow{2}{*}{ Deff } \\
\hline & Sim & $\%$ & Não & $\%$ & & \\
\hline Trabalho & 1.250 & 62,5 & 1.952 & 29,8 & $(1.621-4.783)$ & 1,9 \\
\hline Estudo & 543 & 27,1 & 2.588 & 39,6 & $(676-5.586)$ & 3,0 \\
\hline Família & 116 & 5,8 & 1.497 & 22,9 & $(573-2.653)$ & 1,6 \\
\hline Vontade própria & 37 & 1,8 & 390 & 6,0 & $(-204-1.057)$ & 1,7 \\
\hline Outros & 56 & 2,8 & 115 & 1,8 & $(-79-420)$ & 1,0 \\
\hline Total & 2.002 & 100,0 & 6.542 & 100,0 & Qui-quadrado $=$ & $7,18 \mathrm{E}+05^{* * *}$ \\
\hline \multirow{2}{*}{ Destino } & \multicolumn{4}{|c|}{ Envio de remessa } & \multirow{2}{*}{ I. C - $95 \%$} & \multirow{2}{*}{ Deff } \\
\hline & Sim & $\%$ & Não & $\%$ & & \\
\hline Seridó & 0 & 0,0 & 734 & 11,8 & $(330-1.457)$ & 1,093 \\
\hline Outros municípios RN & 499 & 24,5 & 458 & 7,3 & $(95-1.958)$ & 1,701 \\
\hline Outros municípios NE & 802 & 39,4 & 4.263 & 68,3 & $(2.905-7.689)$ & 2,833 \\
\hline Outras regiões & 734 & 36,0 & 786 & 12,6 & $(433-3.002)$ & 1,897 \\
\hline Total & 2.035 & 100,0 & 6.241 & 100,0 & Qui-quadrado = & $8,56 \mathrm{E}+5^{* * *}$ \\
\hline
\end{tabular}

Fonte: Survey Seridó Potiguar, 2017. ${ }^{* *} \mathrm{p}<0,01,{ }^{*} \mathrm{p}<0,05, * \mathrm{p}<0,1$ para teste $X$-squared de diferenças entre proporções.

Outras fontes de renda que exercem um papel relevante na região são os programas de transferência de renda. Dentre os programas, os mais mencionados pelos entrevistados, e que também constituem em transferências monetárias para os domicílios do Seridó, destacaram-se o Programa Bolsa Família (PBF), o Benefício de Prestação Continuada (BPC) e o Auxílio Doença. Em 55,4\% dos domicílios sem emigrante não há beneficiário de qualquer programa social do governo contra 61,8\% dos domicílios com emigrante, assim como mostra a Tabela 4. A participação de domicílios beneficiários do PBF em domicílios sem emigrante $(20,2 \%)$ é superior ao mesmo percentual em domicílios com emigrante $(17,4 \%)$. No entanto, nos domicílios com emigrante os percentuais de domicílios que recebem o BPC $(3,1 \%)$ e o auxílio doença $(4,7 \%)$ são superiores em relação aos mesmos percentuais para domicílios sem emigrante $(2,1 \%$ e $0,6 \%$, respectivamente). No entanto, o efeito do desenho amostral complexo foi considerável, com a estatística Deff se distanciando um pouco da unidade, o que dá pouca consistência às estimativas. Entretanto, 
o teste Qui-quadrado foi satisfeito, confirmando diferenças estatisticamente significativas entre essas proporções.

Tabela 4 - Seridó Potiguar: distribuição relativa (\%) dos domicílios urbanos, com e sem emigrantes, segundo o recebimento de benefício social, 2017

\begin{tabular}{|c|c|c|c|c|c|c|}
\hline \multirow{3}{*}{ Variável } & \multicolumn{4}{|c|}{ Domicílios } & \multirow{3}{*}{ I. C- $95 \%$} & \multirow{3}{*}{ Deff } \\
\hline & \multicolumn{2}{|c|}{ Com emigrante } & \multicolumn{2}{|c|}{ Sem emigrante } & & \\
\hline & $\mathbf{N}$ & $\%$ & $\mathbf{N}$ & $\%$ & & \\
\hline Benefício Social & - & - & - & - & - & - \\
\hline Nenhum & 5.741 & 61,8 & 27.674 & 55,4 & (23.619-43.211) & 4,5 \\
\hline Bolsa Família & 1.619 & 17,4 & 10.119 & 20,2 & (8.773-14.704) & 1,7 \\
\hline BPC & 288 & 3,1 & 1.041 & 2,1 & $(-221-2.878)$ & 2,4 \\
\hline Bolsa Estiagem & 33 & 0,4 & 390 & 0,8 & $(-500-1.344)$ & 2,5 \\
\hline Seguro desemprego & 96 & 1,0 & 73 & 0,1 & $(-232-570)$ & 1,7 \\
\hline Auxílio doença & 434 & 4,7 & 318 & 0,6 & $(-127-1.630)$ & 1,8 \\
\hline Auxílio defeso & 46 & 0,5 & 0 & 0,0 & $(-66-158)$ & 0,9 \\
\hline Outros & 73 & 0,8 & 611 & 1,2 & $(-199-1.566)$ & 1,9 \\
\hline Não respondeu & 956 & 10,3 & 9.757 & 19,5 & (4.309-17.118) & 3,8 \\
\hline Total & 9.286 & 100,0 & 49.983 & 100,0 & Qui-quadrado = & $1,88 \mathrm{E}+06 * * *$ \\
\hline $\begin{array}{l}\text { Alguém teria } \\
\text { migrado na ausência } \\
\text { do benefício social? }\end{array}$ & - & - & - & - & - & - \\
\hline Sim & 266 & 7,5 & 2.347 & 10,5 & (823-4.402) & 2,0 \\
\hline Não & 2.307 & 65,1 & 11.177 & 50,1 & $(10.668-16.300)$ & 1,5 \\
\hline $\begin{array}{l}\text { Não sabe/ não } \\
\text { respondeu }\end{array}$ & 971 & 27,4 & 8.785 & 39,4 & $(3.222-16.291)$ & 4,0 \\
\hline Total & 3.544 & 100,0 & 22.309 & 100,0 & Qui-quadrado = & $275,66 * * *$ \\
\hline
\end{tabular}

Fonte: Survey Seridó Potiguar, 2017. ${ }^{* * *} \mathrm{p}<0,01,{ }^{*} \mathrm{p}<0,05,{ }^{*} \mathrm{p}<0,1$ para teste $X$-squared de diferenças entre proporções. $\mathrm{n}=\mathrm{n}$ 을 de observações pequeno.

Os programas sociais nessa região representam um apoio fundamental para os domicílios com emigrante e sem emigrante, embora ainda existam poucas evidências sobre essa afirmação (Ojima, 2013). O PBF, por exemplo, é reportado em alguns estudos como uma política que contribui para que a população permaneça no lugar de residência (Araújo, 2012; Gama, 2012). Por outro lado, esse benefício é destinado às famílias inscritas no Cadastro Único (Cadúnico) e engloba uma parcela da população que dispõe de poucos 
recursos para se mover. A migração seria uma estratégia de sobrevivência que além de uma série de motivações, exige a oportunidade de escolha dos indivíduos e dos domicílios.

Nesse sentido, a migração como estratégia de sobrevivência é impulsionada quando a ineficiência de políticas ou a inexistência delas não permitem que sejam sanadas as ameaças à subsistência da população na região. No caso do Seridó Potiguar essas ameaças são representadas pelas estiagens severas. Isso sublinha a relevância em entender tanto as motivações e circunstâncias dos deslocamentos quanto da necessidade em identificar o acesso a políticas sociais nas regiões de origem (Betts, 2013).

De acordo com Araújo (2012), as políticas sociais foram importantes para manter a população nordestina na região, ao invés de buscar trabalho em outras regiões do país devido às estiagens prolongadas na sua terra natal. Gama (2012) constatou, para o estado de Minas Gerais, que a probabilidade de migrar diminui para beneficiários do PBF enquanto que Ojima, Azevedo e Oliveira (2015), para o Rio Grande do Norte e o Semiárido Potiguar, analisaram que a aquisição da aposentadoria aumenta a chance do indivíduo retornar a sua região de origem. No entanto, em ambos os casos, a migração tem uma perspectiva individual sem considerar os efeitos das políticas sobre a migração no âmbito domiciliar.

O BPC e o auxílio doença envolvem certos segmentos da sociedade, como os idosos, que necessitariam de maiores cuidados e que poderiam ser motivados a migrar em busca de serviços e cuidados com saúde que não são oferecidos na cidade de origem. Por outro lado, como o valor do BPC e do auxílio doença podem exercer uma maior influência sobre o processo migratório, pois com valores maiores, poderiam subsidiar a migração do beneficiário e outros indivíduos. De toda forma, o PBF é de suma importância para as famílias, sendo que para $15,9 \%$ delas era a única fonte de renda do domicílio.

Quando perguntados sobre a possibilidade de algum morador migrar caso a família não recebesse o benefício social, em 10,5\% dos domicílios sem emigrante a resposta foi afirmativa, corroborando com a literatura que diz que esses benefícios são importantes para manter a população na sua região (Araújo, 2012; Correia, Ojima, 2018). Para os domicílios com emigrante essa proporção foi de apenas 7,5\%, o que significa dizer que na percepção da pessoa de referência do domicílio os indivíduos desses domicílios teriam emigrado independentemente da existência do recebimento do benefício do programa social.

As respostas a essa pergunta se traduzem, contudo, em resultados diferentes quando feita para domicílios com emigrante e domicílios sem emigrante. 
Neste último, a pessoa de referência respondeu majoritariamente (50,1\%) que ninguém teria migrado na ausência do benefício social. Mas na maior parte dos domicílios com emigrante $(65,1 \%)$, a pessoa de referência respondeu que "não" quando perguntada se "alguém teria migrado na ausência do benefício social?", reafirmando a relevância dos programas sociais para a migração dos indivíduos desses domicílios. Certamente, moradores dos domicílios com emigrante e que no caso estiveram mais próximos da experiência migratória podem fornecer resultados mais consistentes em relação à relevância que os programas sociais teriam na decisão do processo migratório.

Esses resultados sinalizam consonância com a literatura sobre o tema, em que ter acesso a transferências monetárias em contextos de secas aumentam as chances de mobilidade, especialmente para os indivíduos do sexo masculino (Muller et al., 2019). Porém, pelos dados coletados não é possível entender como as transferências monetárias podem ser importantes para explicar a mobilidade como estratégia de adaptação às secas por sexo no Seridó Potiguar, permanecendo como um desafio para as investigações futuras.

\section{Desafios para as políticas de adaptação e de planejamento urbano e regional no Seridó Potiguar}

A emigração do Seridó tem um significado positivo à medida que as remessas para as famílias na região de origem são incorporadas em sua economia, servindo de suporte para as famílias especialmente em determinadas situações de vulnerabilidade - como é o caso dos períodos de seca. Porém, isso representa uma dependência dos municípios de origem da migração e sinaliza desafios para regiões receptoras, havendo, assim, uma maior necessidade de planejamento e capacidade orçamentária para gerenciar a demanda por serviços básicos de educação, saúde e também de acesso ao mercado de trabalho para que retenha essa mão de obra.

A urbanização do semiárido nordestino, embora tenha suas singularidades, pode oportunizar o engajamento das famílias em outras atividades econômicas quanto um meio de gerenciar com maior eficiência a demanda por serviços básicos e de educação, saúde e abastecimento de água nas cidades. A migração e a concentração de pessoas nas áreas urbanas pode ser importante para enfrentar os desafios ambientais, desde que sejam acompanhadas por políticas adequadas de planejamento urbano. As políticas devem considerar estes impactos para evitar que formas de ocupação excludentes se reproduzam e maximizem os benefícios que essas estratégias ofereceriam.

As análises efetuadas, no entanto, não permitem afirmar até que ponto essas transferências, especialmente as remessas de emigrantes, podem intensificar processos de exploração e degradação do meio ambiente, seguindo 
a mesma lógica do contexto amazônico observada em estudos citados nesse trabalho. Para isso, seria necessário conduzir estudos específicos considerando as singularidades de uma região onde as estratégias domiciliares aparentam maior complexidade em relação ao contexto amazônico devido a um sistema social e econômico mais diversificado, dependente do clima e urbanizado.

Por fim, esse artigo apresenta uma análise inicial relevante para explorar mais detalhadamente os motivos da migração no interior do Nordeste e o papel das transferências monetárias para a adaptação dos domicílios às secas. Nesse sentido, espera-se desenvolver outras pesquisas que possam ampliar a compreensão das especificidades da região relacionadas à mobilidade e outros fenômenos no contexto das secas. Inclusive entender até que ponto essas transferências monetárias contribuem para o bem-estar das famílias e funcionam como uma estratégia de sobrevivência, especialmente comprar água e outros meios de subsistência; ou de que modo esses recursos são introduzidos na economia da região.

\footnotetext{
Agradecimentos. Os autores agradecem suporte financeiro à pesquisa que originou esse artigo, através da Rede Brasileira de Pesquisas em Mudanças Climáticas (Rede Clima, FINEP/MCTI) e do Conselho Nacional de Desenvolvimento Científico e Tecnológico (CNPq), 447688/2014-6 e 306567/2016-4. Também agradecemos a agência de fomento Coordenação de Aperfeiçoamento de Pessoal de Nível Superior (Capes/PROEX) pela bolsa de doutorado.
}

\section{Referências bibliográficas}

ADGER, W. Neil et al. Focus on environmental risks and migration: Causes and consequences. Environ. Res. Lett., v. 10, n. 1, 060201, 2015.

ARAÚJO, Tania Barcelar. Economia do semiárido nordestino: a crise como oportunidade. Revista Coletiva, v. 16, n. 1, 2012.

BAENINGER, Rosana. Rotatividade Migratória: um novo olhar para as migrações internas no Brasil. REMHU, Revista Interdisciplinar da Mobilidade Humana, v. 20, n. 39, p. 77-100, 2012.

BARBIERI, Alisson F. Mudanças climáticas, mobilidade populacional e cenários de vulnerabilidade para o Brasil. REMHU, Revista Interdisciplinar da Mobilidade Humana, v. 19, n. 36, p. 95-112, 2011.

BARBIERI, Alisson F.; CARR, David L.; BILSBORROW, Richard E. Migration within the frontier: the second generation colonization in the Ecuadorian Amazon. Popul. Res. Policy Rev., v. 28, n.3, p. 291-320, 2009.

BARBIERI, Alisson F. et al. Climate change and population migration in Brazil's Northeast: scenarios for 2025-2050. Popul. Environ., v. 31, p. 344-370, 2010.

BARBIERI, Alisson F. et al. Mobility as a livelihood adaptation in the Brazilian semiarid. In: International Conference Migrations, Environment and Climate: What risks Inequalities? Annals of... Paris, France: INED, 2018. 
BARBIERI, Alisson F.; PAN, William K. People, land, and context: multilevel determinants of off-farm employment in the Ecuadorian Amazon. Popul. Space Place, v. 19, n. 5, p. 558-579, 2013.

BARDSLEY, Douglas K.; HUGO, Graeme. Migration and climate change: examining thresholds of change to guide effective adaptation in decision-making. Popul. Environ., v. 32, n. 2/3, p. 238-262, 2010.

BETTS, Alexander. State fragility, refugee status and 'survival migration'. Forced Migr. Rev., n. 43, p. 4-6, 2013.

BLACK, Richard et al. The effect of environmental change on human migration. Glob. Environ. Change, v. 21, supl. 1, p. S3-s11, 2011.

BOURDIEU, Pierre. The forms of capital. In: RICHARDSON, John G. (org.). Handbook of Theory and Research for the Sociology of Education. Nova Iorque: Greenwood, 1985, p. 24-58.

CAMERON, A. Colin; TRIVEDI, Pravin K. Microeconometrics: methods and applications. New York: Cambridge University Press, 2005.

CARR, David L. et al. Un análisis multinivel de población y deforestación en el Parque Nacional Sierra de Lacandón (Petén, Guatemala). Doc. Anál. Geogr., v. 52, p. 49-67, 2008.

CORBIN, Hisakhana P.; ARAGÓN, Luis E. Imigração e garimpo, emigração e remessas: dois pilares da economia da Guiana. Territórios \& Fronteiras, v. 8, p. 66-88, 2015.

CORREIA, Isac; OJIMA, Ricardo. Composição demográfica domiciliar e (i) mobilidade no Seridó Potiguar: vulnerabilidade à seca e estratégias domiciliares no sertão nordestino. Territórios \& Fronteiras, v. 11, n. 1, p. 199-223, 2018.

CORREIA, Isac. Vulnerabilidade e adaptação no Seridó Potiguar: a (i)mobilidade e estratégias domiciliares. (Dissertação de Mestrado em Demografia). Departamento de Demografia e Ciências Atuariais - DDCA/UFRN. Natal/RN: UFRN, 2018.

DUO, Yue et al. Impacts of cash transfer programs on rural livelihoods: a case study in the Brazilian Amazon Estuary. Hum. Ecol., v. 45, n. 5, p. 697-710, 2017.

FURTADO, Celso. A operação Nordeste. Rio de Janeiro: Ministério da Educação e Cultura, Instituto Superior de Estudos Brasileiros, 1959.

FUSCO, Wilson; DUARTE, R. Regiões metropolitanas do Nordeste: origens, destinos e retorno dos migrantes. In: XVII Encontro Nacional sobre Migrações. Anais... Caxambu/MG: ABEP, 2010.

GAMA, Luiz C. D. O Programa Bolsa Família pode influenciar a decisão por migrar? Uma análise para o estado de Minas Gerais. In: XV Seminário sobre a Economia Mineira. Anais... Diamantina/MG, 2012.

GILBERT, Genevieve; MCLEMAN, Robert. Households access to capital and its effects on drought adaptation and migration: a case study of rural Alberta in the 1930s. Popul. Environ., v. 32, p. 3-26, 2010.

GRAY, Clark; MUELLER, Valerie. Drought and population mobility in rural Ethiopia. World Development, v. 40, n. 1, p. 134-145, 2012. 
HUGO, Graeme. Environmental concerns and international migration. Int. Migr. Rev., v. 30, n. 1, p. 105-131, 1996.

KHAN, A. Saaed et al. Efeito da seca sobre a produção, a renda e o emprego agrícola na microregião geográfica de Brejo Santo e no Estado do Ceara. Revista Econômica do Nordeste, v. 36, n. 2, p. 242-262, 2005.

KOUBI, Vally; STOLL, Sebastian; SPILKER, Gabriele. Perceptions of environmental change and migration decisions. Climatic Change, v. 138, p. 439-451, 2016.

LEE, Ewerert. Uma teoria sobre a migração. In: MOURA, Hélio Augusto de (org.). Migração interna, textos selecionados. Fortaleza: BNB/ETENE, 1980, p. 89-114, 1966.

LOMBARDI, Thaís T.; GUEDES, Gilvan R.; BARBIERI, Alisson F. As estratégias de sobrevivência nos estudos sobre a fronteira na Amazônia: contribuições das perspectivas rurais e urbanas. Territórios \& Fronteiras, v. 8, n. 2, 2015.

MARTINE, George. A redistribuição espacial da população brasileira durante a década de 80. Texto para Discussão. Brasília: IPEA, 1994.

MASSEY, Douglas; AXINN, William G.; GHIMIRE, Dirgha J. Environmental change and out-migration: evidence from Nepal. Popul. Environ., v. 32, p. 109-136, 2010.

MELO NETO, João Cabral. Morte e Vida Severina. São Paulo: Teatro da Universidade Católica, 1965.

MONTE-MÓR, Roberto L. O que é o urbano no mundo contemporâneo. Texto para Discussão. Belo Horizonte, Cedeplar/UFMG, n. 281, 2006.

MULLER, Valerie et al. Do social protection programs foster short-term and longterm migration adaptation strategies? Environ. Dev. Econ., p. 1-24, 2019.

NAWROTZKI, Raphael J.; DEWAARD, Jack. Climate shocks and the timing of migration from Mexico. Popul. Environ., v. 38, p. 72-100, 2016.

OJIMA, Ricardo. Urbanização, dinâmica migratória e sustentabilidade no semiárido nordestino: o papel das cidades no processo de adaptação ambiental. Cad. Metrop., v. 15, n. 29, p. 35-54, 2013.

OJIMA, Ricardo; AZEVEDO, Paulo R. M.; OLIVEIRA, Herick C. G. O impacto da aposentadoria no retorno migratório ao Rio Grande do Norte e ao semiárido potiguar. Informe Gepec, v. 19, n. 1, p. 6-19, 2015.

OLIVEIRA, Kleber F. Demografia da pobreza em Sergipe: migração, renda e características municipais a partir dos anos 1980. (Tese de Doutorado em Demografia). Universidade Estadual de Campinas (UNICAMP). Campinas/SP: UNICAMP, 2008.

PEARSON, Karl. On the criterion that a given system of deviations from the probable in the case of a correlated system of variables is such that it can be reasonably supposed to have arisen from random sampling. Philosophical Magazine, v. 50, n. 302, p. 157-175, 1990.

QUEIROZ, Raquel. O Quinze. Rio de Janeiro: José Olympio, 1930. 
RAMOS, Graciliano. Vidas secas. Rio de Janeiro: José Olympio, 1938.

RN - GOVERNO DO ESTADO DO RIO GRANDE DO NORTE. Decreto $n^{\circ} 27.315$ de 18 de setembro de 2017. Diário Oficial, 18 set. 2017.

SHERBININ, Alex et al. Rural household demographics, livelihoods and the environment. Glob. Environ. Change, v. 18, n. 1, p. 38-53, 2008.

SOUZA, Everaldo B. et al. Cap. 9: Mudanças ambientais de curto e longo prazo: projeções e atribuição. In: AMBRIZZI, Tércio; ARAUJO, Moacyr (org.). Base científica das mudanças climáticas. Contribuição do Grupo de Trabalho 1 do Painel Brasileiro de Mudanças Climáticas ao Primeiro Relatório da Avaliação Nacional sobre Mudanças Climáticas. Rio de Janeiro: COPPE/UFRJ, v. 1, p. 320346, 2014.

VANWEY, Leah K.; D’ANTONA, Alvaro O.; BRONDÍZIO, Eduardo S. Household demographic change and land use/land cover change in the Brazilian Amazon. Popul. Environ., v. 28, n. 3, p. 163-185, 2007.

VANWEY, Leah K.; GUEDES, Gilvan R.; D'ANTONA, Alvaro O. Out-migration and land-use change in agricultural frontiers: insights from Altamira settlement project. Popul. Environ., v. 34, n. 1, p. 44-68, 2012.

WARNER, Koko et al. Climate change and migration: reflections on policy needs. MEA Bulletin, Guest Article No. 64, 2009. Disponível em: < http://www.iisd.ca/ mea-l/guestarticle64.html>. Acesso em: 02.05.2017. 